Meta

Journal des traducteurs

Translators' Journal

\title{
Noms d'animaux et difficultés de traduction
}

\section{Pierre Avenas et Henriette Walter}

Volume 55, numéro 4, décembre 2010

De la localisation à la délocalisation - le facteur local en traduction

From Localization to Delocalization - The Local Factor in Translation

URI : https://id.erudit.org/iderudit/045690ar

DOI : https://doi.org/10.7202/045690ar

Aller au sommaire du numéro

\section{Éditeur(s)}

Les Presses de l'Université de Montréal

ISSN

0026-0452 (imprimé)

1492-1421 (numérique)

Découvrir la revue

\section{Citer cet article}

Avenas, P. \& Walter, H. (2010). Noms d'animaux et difficultés de traduction. Meta, 55(4), 769-778. https://doi.org/10.7202/045690ar

\section{Résumé de l'article}

La traduction des noms d'animaux doit tenir compte des taxonomies scientifiques, lesquelles évoluent parallèlement aux connaissances des sciences naturelles. On attribue un nom latin scientifique à chaque espèce, mais les noms vernaculaires de la langue commune suivent des dynamiques complexes. Tout d'abord, les noms vernaculaires ne sont pas aussi nombreux que les noms d'espèces. Par ailleurs, les espèces sont parfois confondues, les équivalences dénominatives entre langues peuvent présenter de sérieux problèmes, les faux amis ne sont pas rares, et il faut compter avec les expressions idiomatiques et les variantes régionales. Enfin, l'emploi des noms d'animaux dans les mythes et les oeuvres littéraires ou artistiques s'écarte de la rigueur imposée par la zoologie, en raison de la prédominance de la fonction connotative sur la fonction dénotative. La traduction d'un nom d'animal la mieux adaptée au contexte nécessite donc une approche diversifiée, associant des connaissances de sciences naturelles à des considérations linguistiques.
Ce document est protégé par la loi sur le droit d'auteur. L’utilisation des services d’Érudit (y compris la reproduction) est assujettie à sa politique d'utilisation que vous pouvez consulter en ligne.

https://apropos.erudit.org/fr/usagers/politique-dutilisation/ 


\title{
Noms d'animaux et difficultés de traduction
}

\author{
PIERRE AVENAS \\ Paris, France \\ pier.avenas@orange.fr
}

HENRIETTE WALTER

Université Rennes 2 - Haute Bretagne, Rennes, France

henriettewalter@neuf.fr

\begin{abstract}
RÉSUMÉ
La traduction des noms d'animaux doit tenir compte des taxonomies scientifiques, lesquelles évoluent parallèlement aux connaissances des sciences naturelles. On attribue un nom latin scientifique à chaque espèce, mais les noms vernaculaires de la langue commune suivent des dynamiques complexes. Tout d'abord, les noms vernaculaires ne sont pas aussi nombreux que les noms d'espèces. Par ailleurs, les espèces sont parfois confondues, les équivalences dénominatives entre langues peuvent présenter de sérieux problèmes, les faux amis ne sont pas rares, et il faut compter avec les expressions idiomatiques et les variantes régionales. Enfin, l'emploi des noms d'animaux dans les mythes et les œuvres littéraires ou artistiques s'écarte de la rigueur imposée par la zoologie, en raison de la prédominance de la fonction connotative sur la fonction dénotative. La traduction d'un nom d'animal la mieux adaptée au contexte nécessite donc une approche diversifiée, associant des connaissances de sciences naturelles à des considérations linguistiques.
\end{abstract}

\section{ABSTRACT}

The translation of animal names must take into account scientific taxonomies, which evolve together with the development of natural sciences. Each species is given a scientific Latin name, but vernacular names in common language follow complex dynamics. Moreover, confusion may crop up between different species, denominative equivalences between languages may raise serious problems, "false friends" are not rare, and one must take into account idiomatic phrases and regional variants. Finally, the way in which animal names are used in myths and in literary and artistic works diverge from the exactness imposed by zoology, due to the predominance of connotative function over denotative function. As a conclusion, we can say that the best translation of an animal name in such or such context necessitates a diversified approach, combining natural sciences knowledge and linguistic considerations.

\section{MOTS-CLÉS/KEYWORDS}

taxonomie scientifique, nom vernaculaire, équivalence dénominative scientific taxonomy, vernacular name, naming equivalence

Parmi les différents problèmes qui se posent au traducteur, le choix des noms d'animaux à employer dans la langue cible est très délicat, car il doit obligatoirement tenir compte des taxonomies fixées par les naturalistes. Toutefois, les noms vernaculaires abondent et leur dynamique est très variable d'une langue à l'autre. Devant cette abondance de noms souvent mal maîtrisée, le traducteur doit choisir entre la rigueur reflétant les règles de la zoologie qu'impose la traduction d'un texte spécialisé (scientifique, technique ou même juridique), et la souplesse de traduction, mieux adaptée 
à un texte littéraire ou au grand public, quitte à s'écarter, en toute connaissance de cause, de la précision scientifique.

\section{La classification binominale des êtres vivants : des taxonomies en évolution}

Pour donner une solution concertée et définitive à la difficulté de l'identification des espèces, les naturalistes du monde entier s'accordent, depuis la $10^{\mathrm{e}}$ édition du Systema Naturae de Linné en $1758^{1}$, sur une dénomination officielle binominale, en latin, des plantes et des animaux: le nom scientifique du chat domestique, par exemple, est Felis catus. Ainsi, tous les spécialistes de zoologie du monde comprennent que ce mammifère appartient au genre Felis, et, au sein de ce genre, à l'espèce catus.

Ce latin scientifique est un véritable espéranto pour les naturalistes: partout dans le monde, l'emploi des noms latins scientifiques garantit la référence à une même espèce. Mais la question se complique avec les sous-espèces, appelées aussi variétés ou races, pour lesquelles on ajoute un troisième terme latin: ainsi le loup gris, sauvage, est nommé Canis lupus lupus, alors que le chien, domestique, est Canis lupus familiaris.

Le latin des naturalistes est une langue un peu particulière (Walter 2005), qui utilise, certes, des noms du latin classique ou du latin plus tardif, mais aussi des noms entièrement fabriqués. Ainsi, felis est bien le nom du chat sauvage en latin classique, et cattus est celui du chat domestique en bas-latin, avec tout de même ici une particularité orthographique, due à Linné, qui a écrit catus avec un seul $<\mathrm{t}>$. En revanche, l'un des lémuriens les plus étranges qui soit, avec ses doigts démesurément allongés, l'aye-aye, a un nom scientifique, Daubentonia madagascariensis, que l'on cherchera vainement dans la référence en matière de dictionnaire latin-français, le dictionnaire de Gaffiot $(1934)^{2}$. De fait, le nom de cette espèce honore le naturaliste Daubenton et rappelle que l'animal vit dans l'île de Madagascar.

Dans certains cas, l'origine et le sens de certains noms latins scientifiques restent inconnus, car les premiers naturalistes, dont Linné lui-même, ont bien souvent créé des noms sans les expliquer. Aujourd'hui, cette pratique n'est plus admise et les naturalistes, lorsqu'ils baptisent de nouvelles espèces, doivent justifier et expliquer les noms qu'ils proposent à la communauté scientifique internationale, qui les enregistre officiellement.

La classification du monde vivant est constamment revue et augmentée en fonction des découvertes de nouvelles espèces et des progrès de la phylogénétique, qui apporte des précisions sur la parenté qui existe entre les espèces. Cela peut conduire à des difficultés car, si les noms d'espèce sont toujours conservés, les noms de genre, en revanche, peuvent être changés au cours des révisions de la classification. Ainsi, pour le lion, on peut encore trouver dans les livres un peu anciens le nom Felis leo (retenu par Linné en 1758), alors que son nom latin scientifique officiellement entériné aujourd'hui est Panthera leo.

Une liste officielle de référence, aussi récente que possible et reconnue universellement, est donc indispensable. Il est ainsi possible de se référer, aujourd'hui, à la classification de Wilson et Reeder (2005), qui recense environ 5500 espèces de mammifères, à celle de Howard et Moore (Dickinson 2003) pour environ 10000 espèces d'oiseaux et à celle de Nelson (2006) pour près de 30000 espèces de poissons. De 
plus, des sites Internet sont accessibles, comme le site Fishbase , $^{3}$ validé et actualisé en permanence par les ichtyologues du monde entier.

Le recours à ces dénominations, précises et universelles, est essentiel dans la production de textes à caractère scientifique et technique ou juridique, notamment lorsqu'il s'agit de protection d'espèces menacées ou en danger d'extinction, ou de réglementation de la chasse et de la pêche. Il faut d'ailleurs souligner que le recours aux noms latins scientifiques prend une importance croissante en ichtyologie, en raison de la surpêche qui met en danger certaines espèces : ainsi, le thon rouge - c'està-dire à chair rouge - s'appelle en anglais blue-fin tuna (lit., thon à aileron bleu). Il porte de plus des noms différents dans d'autres langues, mais son nom scientifique est unique: Thunnus thynnus. Ce qui est nouveau, c'est que les emballages des poissons surgelés ou en conserve mentionnent de plus en plus souvent le nom latin scientifique de l'espèce. Finalement, c'est par les poissons que le grand public se trouve être le plus directement en contact avec le latin des naturalistes, bien que les amateurs de jardinage le soient également par les semences et les plantes.

\section{La problématique des noms vernaculaires}

La connaissance de la nomenclature officielle est certes indispensable, mais, dans un contexte de traduction, il est non moins nécessaire de connaître la dynamique des noms vernaculaires. Celle-ci est en fait fort complexe. Nous évoquerons, dans ce qui suit, différents problèmes : la représentation des noms vernaculaires dans les dictionnaires usuels, le problème de la confusion des espèces dans des contextes non scientifiques, la problématique des équivalences dénominatives et des faux amis, la question des expressions idiomatiques et des variantes régionales.

\subsection{Les noms vernaculaires dans les dictionnaires usuels}

Une première constatation s'impose: un seul nom vernaculaire sert d'hyperonyme à de nombreux noms spécifiques. Ainsi, le nom chauve-souris recouvre en fait environ 1200 espèces différentes de mammifères volants (soit $20 \%$ des espèces de mammifères connus!), celui d'oiseau-mouche (ou colibri) plus de 330 espèces et celui de poisson-chat (parfois appelé aussi silure) près de 3000 espèces dans le monde. Ce phénomène se traduit par la présence, en français courant (représenté par le lexique consigné dans les dictionnaires d'usage Hachette, Petit Larousse et Petit Robert), de seulement un peu moins de 300 noms de mammifères (Walter et Avenas 2003), 300 noms d'oiseaux (Walter et Avenas 2007), et 200 noms de poissons (Walter et Avenas, à paraître $\left.{ }^{4}\right)$. Ces noms sont parfois reliés par des relations d'hyperonymie, ce qui permet de moduler la précision de la désignation en contexte: ainsi, le morillon, qui est un canard sauvage dont le mâle, presque entièrement noir, est huppé, fait partie des fuligules, qui eux-mêmes font partie des canards.

\subsection{Confusion dans les espèces}

Il faut tout d'abord remarquer que la langue commune ne tient pas toujours compte de la réalité zoologique. On sait par exemple que le chameau a deux bosses et que le dromadaire n'en a qu'une, mais il est fréquent d'entendre le commun des mortels 
parler de chameau, de chamelle et de chamelier dans les deux cas. Un autre exemple est fourni, en français, par l'usage du nom corbeau, qui s'emploie très souvent à la place de corneille (cet oiseau est très présent en région parisienne, où on ne trouve pas de vrais corbeaux). En toute rigueur, l'anglais raven correspond à corbeau, alors que crow se traduit par corneille. Or, crow s'emploie couramment pour les deux espèces. Dès lors, on traduira souvent crow par corbeau, sans trop se soucier de la distinction zoologique entre corneille et corbeau, souvent oubliée, en France comme en Angleterre. Cette confusion est entretenue par l'imprécision des dictionnaires bilingues généraux. Ainsi, le dictionnaire bilingue Robert \& Collins Super Senior $(1995)^{5}$ propose les «équivalences» suivantes:

- crow n (Orn) corneille f; (generic term) corbeau

- corbeau nm (terme générique) crow - (grand) corbeau raven - corbeau freux rook - corbeau corneille crow

On rencontre la même situation avec la mouette, souvent confondue avec le goéland. Ces oiseaux se ressemblent, mais appartiennent clairement à des espèces différentes: les goélands sont de grande taille - avec une envergure dépassant souvent le mètre - alors que les mouettes sont plus petites. Mais il n'y a guère qu'en français qu'il existe deux noms vernaculaires: mouette est d'origine germanique, et goéland vient du breton goelañ «pleurer» (on dit d'ailleurs que le goéland pleure, mais on notera qu'une espèce de mouette est dite «rieuse» [Larus ridibundus]). Dans la plupart des autres langues d'Europe de l'Ouest, un seul nom existe, comme (sea)gull en anglais: il faudra donc se contenter de large gull et small gull si l'on veut distinguer le goéland de la mouette dans une traduction vers l'anglais. Et, dans le sens inverse, de l'anglais en français, pour traduire gull, il faudra tenir compte du contexte: dans Les oiseaux d'Hitchcock, ce sont en vérité des goélands mais le nom mouette pourrait lui être préféré, si le traducteur souhaite utiliser un nom plus connu.

Nous verrons plus en détail, dans la section 3, la problématique des noms d'animaux en rapport avec les œuvres que l'on pourrait qualifier de religieuses, voire mythiques, comme le Livre de Jonas, ou littéraires.

\subsection{Quelques problèmes d'équivalences dénominatives}

Le passage d'une langue à l'autre est l'occasion de mettre en relief certains problèmes d'équivalences dénominatives: les noms peuvent évoquer des caractéristiques différentes d'un animal, certaines dénominations peuvent tout simplement être absentes ou, au contraire, une pléthore de dénominations peut être observée.

Dans le premier cas, le nom vernaculaire a un pouvoir évocateur particulier dans une langue donnée. Celui-ci n'est toutefois pas retrouvé dans les dénominations utilisées dans d'autres langues, lesquelles emploient plutôt un nom complexe constitué d'un hyperonyme associé à un qualificatif. Ainsi, une mouette possède un nom, en anglais, qui est une pure onomatopée évoquant son cri, kittiwake. Le nom français, mouette tridactyle, qui est plutôt une appellation savante, évoque une autre caractéristique, c'est-à-dire le fait de posséder trois doigts au lieu de quatre comme chez les autres mouettes. Il n'a toutefois plus le caractère pittoresque du nom anglais. Autre exemple, on voit voleter, le soir, des petites chauves-souris, appelées du joli nom de pipistrelle en français. Ce nom a été repris intégralement en anglais (pipistrelle), mais 
pas par d'autres langues européennes, dans lesquelles on se contentera de la traduction littérale de petite chauve-souris, ce qui n'est évidemment que partiellement satisfaisant. Dernier exemple, les oiseaux nommés de façon univoque par warbler (c'est-à-dire "gazouilleur») en anglais, appartiennent à plusieurs espèces désignées en français par une série de noms différents: fauvette, rousserole (effarvate), pouillot, phragmite...

\subsection{Les faux amis}

Il faut se méfier de certains faux amis, tels que, par exemple, puffin, qui est le nom anglais pour macareux en français, et shearwater, qui est le nom anglais pour puffin. Le puffin le plus commun (Puffinus puffinus) est appelé en français Puffin des Anglais et en anglais Manx shearwater, c'est-à-dire, littéralement, "puffin de l'île de Man». Cet usage atypique du nom puffin en anglais ne s'explique que par une confusion entre les deux oiseaux de mer, qui se côtoient dans les mêmes habitats, même s'ils ne se ressemblent pas vraiment.

Des confusions pires que celle-ci ont pu exister dans le passé: ainsi, le nom russe du chameau, verblûd, signifie étymologiquement "gros éléphant» (Avenas 2005).

Le cas du pingouin est peut-être plus connu: on distingue, en français, le pingouin (de l'hémisphère Nord, qui peut nager et voler) et le manchot (de l'hémisphère Sud, qui peut nager, mais ne peut pas voler). Des films récents à grand succès, comme La marche de l'Empereur (March of the Penguins dans sa version originale) et Happy Feet (qu'on a renoncé à traduire en allemand, en espagnol et en français, sauf au Québec: Les petits pieds du bonheur) ont rendu les manchots particulièrement célèbres. Du point de vue strictement ornithologique, les noms de genre vernaculaires équivalents entre français et anglais sont manchot et penguin, d'une part, et pingouin et auk, d'autre part. Différentes espèces ont des noms vernaculaires spécifiques, comme emperor penguin pour manchot empereur, ou razorbill (auk) pour pingouin torda.

En tout cas, pour un ornithologue, il ne faut surtout pas traduire l'anglais penguin par le français pingouin, même si l'usage de pingouin en français et de penguin en anglais est loin d'être toujours rigoureux. Si le contexte est en effet dénué de toute spécificité, comme dans le cas de jouets en peluche pour les tout-petits, on emploiera plus facilement pingouin, manchot n'ayant pas un usage très fréquent dans la langue commune ou familière. De ce fait, le dictionnaire bilingue Robert \& Collins Super Senior (1995) entérine les abus de langage:

- pingouin nm: [arctique] auk; (emploi gén) penguin - (petit) pingouin razorbill

- penguin $\mathrm{n}$ : (Orn) manchot $\mathrm{m}$; (general incorrect usage) pingouin $\mathrm{m}$

\subsection{Expressions idiomatiques et variantes régionales}

Le nom de certains animaux apparaît dans des expressions idiomatiques, qui sont rendues de façon variable dans différentes langues sans nécessairement tenir compte de la zoologie. Ainsi, en espagnol, il y a plusieurs variantes pour rendre le nom de l'oie dans plusieurs expressions: ganso, gansa (pour los gansos del Capitolio [les oies $d u$ Capitole]), ánsar (notamment en ornithologie) et oca (pour el juego de la oca [le jeu de l'oie]). En italien, l'expression imagée pelle d'oca (littéralement peau d'oie) se 
traduit en français par chair de poule tandis que collo d'oca (littéralement cou d'oie) se traduit par col de cygne.

L'existence de variantes régionales constitue un des problèmes les plus délicats. Il faut ainsi tenir compte des différences entre l'anglais britannique et l'anglais d'Amérique: le coq s'appelle cock en Grande-Bretagne, mais, pour éviter tout malentendu avec une signification familière (voire vulgaire...), rooster aux États-Unis. D’autre part, l'anglais buffalo désigne le buffle en Europe, mais le bison en Amérique.

De même, en français du Canada, l'élan se dit orignal, le glouton, carcajou (cf. Le Carcajou, de Bernard Clavel). L'outarde se dit bernache, alors que l'oiseau connu en Europe sous le nom de bernache n'a pas de nom particulier au Canada et se nomme oie.

Enfin, en ichtyologie, le bar (Dicentrarchus labrax), par ailleurs fort apprécié des gastronomes, a pour noms équivalents Barsch en allemand et bass en anglais. Mais on sait bien que ce poisson, en français, s'appelle bar dans le nord du pays et loup dans le sud, en particulier s'il vient de la Méditerranée. Les autres langues ne font pas cette distinction purement française et il faudrait donc donner une précision géographique si l'on voulait différencier loup de bar dans une traduction. En fait, d'autres langues ont également plusieurs noms pour ce même poisson: par exemple, en italien, spigola (plutôt au nord) et branzino (plutôt au sud), alors qu'il s'agit souvent d'un poisson de la Méditerranée dans les deux cas. De même, en espagnol, róbalo et lubina (qui sont d'ailleurs des doublets, issus du latin lupus [loup], róbalo dérivant de lobarro par métathèse).

Il ne faudra cependant pas confondre cette espèce avec le loup de mer (Anarhichas lupus), un autre poisson de plus en plus présent dans les poissonneries. C'est un exemple montrant que le nom scientifique permet d'éviter une confusion qui pourrait être entretenue par les noms vernaculaires.

Relevons un dernier cas de variantes propres au français: palombe et pigeon ramier. Il s'agit bien d'une seule et même espèce (Columba palumbus), à l'origine migratrice, maintenant partiellement sédentarisée, notamment dans les villes, à Paris par exemple. Selon le contexte, on emploiera donc l'une ou l'autre des appellations. Ainsi, la chasse à la palombe reste populaire dans le sud-ouest de la France, lorsque ces oiseaux passent en rangs serrés lors de leurs migrations, au grand dam des défenseurs de la nature. En ville, par contre, on parlera plutôt de pigeon ramier, ainsi appelé parce qu'il perche volontiers dans les ramures des arbres, contrairement au pigeon domestique, qui est une sous-espèce du pigeon biset, que l'on voit plus souvent sur les trottoirs et les bâtiments.

\section{Mythes et ouvres littéraires ou artistiques}

Une des confusions les plus connues est celle de la baleine, qui semble habiter l'imaginaire de manière plus puissante que le cachalot ou le requin, avec lesquels elle est parfois confondue. Ainsi, dans le roman Moby-Dick, or The Whale de Herman Melville, Moby Dick, la «baleine blanche», n'est pas une baleine, mais bel et bien un cachalot.

Le monstre du Livre de Jonas n'était pas une baleine non plus! Les naturalistes ont très tôt contesté la traduction du nom de ce monstre marin par baleine. Ce fut le cas en particulier de Guillaume Rondelet, un savant considéré comme le père de 
l'ichtyologie, dans son Histoire entière des poissons parue en $1558^{6}$. Pour lui, ce monstre ne pouvait être qu'un grand requin blanc, qu'il désigne par lamie:

i'ai penfé que ceftoit vne Lamie dans le ventre de la quelle Ionas par la prouidence diuine fut par l'efpace de trois iours, d'où en fortit fain é fauue j’ai pensé que c'était une Lamie dans le ventre de laquelle Jonas resta, par la providence divine, pendant trois jours, et d'où il sortit sain et sauf

(Rondelet 1558: 306; transcription des auteurs)

Et l'auteur, pris d'un scrupule religieux, d'ajouter:

ce qui n'eft aucunement contre la Sainte efcriture: car il eft efcrit que Ionas fut au vẽtre d'vn Cete lequel nom eft general à tous poifsõs fort grands ce qui n'est aucunement contre la Sainte Écriture car il est écrit que Jonas resta dans le ventre d'un Cete dont le nom s'applique à tous les poissons très grands

(Rondelet 1558: 306; transcription des auteurs)

Ici, le terme Cete, employé par Rondelet, est intraduisible: il vient du grec kêtos, qui désignait tout animal marin gigantesque, réel ou imaginaire, par l'intermédiaire du latin cetus, dont le sens s'est restreint pour désigner surtout les baleines et autres cétacés. C'est ainsi qu'en français le terme baleine est devenu traditionnel dans l'expression «Jonas et la baleine». Mais pour Linné lui-même, qui a adopté le terme Cete pour désigner les mammifères qu'on appelle aujourd'hui les cétacés, Jonas avait bien été avalé par un requin...

Beaucoup plus tard, on retrouve une histoire semblable dans Pinocchio, le conte publié en italien par Collodi en 1883. Or, le monstre marin dans ce conte est nommé en italien pescecane, c'est-à-dire «requin». Il semble que Collodi ait bien compris le message des naturalistes ${ }^{7}$ selon lequel le monstre ne pouvait être qu'un requin. Pourtant, cela n'a pas empêché Walt Disney de revenir dans son dessin animé à l'idée d'une baleine, plutôt en forme de cachalot d'ailleurs, et appelée Monstro (un

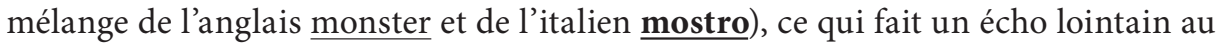
kêtos grec.

La traduction du titre d'une ouvre, écrite ou cinématographique, est parfois délicate. Le roman de Lampedusa, Il Gattopardo (1958), est un cas d'école. Ce titre fait allusion aux armoiries de la famille Salina, où figure un félin tacheté, et, bien sûr, au tempérament fougueux du héros, Fabrizio. Lampedusa a préféré gattopardo à leopardo, qui est le terme héraldique habituel (rappelons ici que le terme héraldique léopard n'a rien de zoologique, puisqu'il désigne en fait un lion regardant de face, par opposition au lion qui est vu de profil), et à ghepardo, qui est le nom zoologique moderne du guépard. Il a choisi un nom un peu vieilli, qui correspond au nom français obsolète chat-pard.

Selon les langues, les traductions du titre du roman ont été différentes:

- en français, le choix s'est porté sur Le Guépard, qui est proche de gattopardo, dont il dérive d'ailleurs étymologiquement, mais qui n’a pas le parfum ancien du titre italien; 
- en anglais, sur The Leopard, ce qui est logique du point de vue de l'héraldique, mais ce nom connote un tempérament plus impitoyable et cruellement prédateur que le nom italien, qui évoque plutôt la débrouillardise et la compromission;

- en espagnol, El Gatopardo est la transposition du titre italien et, en allemand, Der Leopard suit l'anglais.

Aucune de ces traductions n'est parfaite, en tout cas du point de vue zoologique, car un léopard (Panthera pardus) est évidemment très différent d'un guépard (Acinonyx jubatus), dont le nom anglais est cheetah (nom d'origine hindi, qui signifie «tacheté»). Toutefois, le choix, par Lampedusa, du nom gattopardo a eu une répercussion sur la langue italienne, qui emploie aujourd'hui plus facilement le nom gattopardo que le nom officiel de ghepardo.

Ce changement rappelle le cas extraordinaire du renard, qui doit son nom, en français, à un grand succès littéraire. En effet, le renard s'appelait goupil (du latin vulpiculus) au Moyen Âge, et c'est l'immense réussite du Roman de Renart qui est à l'origine du nom renard. Cela entraîne des difficultés de traduction particulièrement aiguës :

- Comment traduire dans une autre langue le nom goupil, certes vieilli, mais toujours présent dans les dictionnaires usuels du français, et encore employé dans des usages littéraires ou plaisants?

- Le titre même du Roman de Renart, s'il est traduit mot à mot, ne contient plus la notion de renard, d'où les adaptations suivantes: Reynard the fox en anglais, Reineke (der) Fuchs en allemand, Romanzo di Renart, ou Romanzo della volpe en italien... et l'espagnol conserve le titre en français.

Les noms d'animaux sont parfois porteurs de connotations étroitement associées aux cultures ou aux croyances populaires, ce dont il faut tenir compte, comme l'illustre la traduction en français du titre d'un film russe: Quand passent les cigognes (film de Kalatozov, Palme d'or à Cannes en 1957). Le titre original russe (Letjat žuravli) signifie, littéralement, "volent les grues», ces oiseaux étant des symboles de longévité et de bonheur dans les pays orientaux. Certes, les grues et les cigognes sont de grands échassiers migrateurs qui ont quelque ressemblance, mais ce sont des oiseaux bien distincts sur le plan zoologique, et qui surtout n'ont pas les mêmes habitudes migratoires. En réalité, cette traduction, qui fait fi de l'ornithologie, vient de ce qu'une traduction littérale (Quand passent les grues) ne saurait connoter l'idée de portebonheur. Par contre, la cigogne a une image positive en Europe de l'Ouest, puisqu'elle annonce «d'heureux évènements» en apportant les bébés. Est-ce sous l'influence du français que les titres du film en italien et en espagnol se réfèrent aussi aux cigognes? L'anglais a par contre adopté une traduction littérale, The cranes are flying, même si l'anglais crane désigne aussi bien l'oiseau que l'engin de levage!

Parmi les noms des grands animaux marins, on trouve un autre exemple de traduction originale avec l'espadon, un poisson bien connu des amateurs de pêche au gros. En français, le nom espadon est largement utilisé, aussi bien sur les marchés que sur les menus des restaurants. Ce nom a d'ailleurs connu un regain de popularité en 1952 lors de la parution en français du Vieil homme et la mer, d'Hemingway: l'histoire raconte en effet la lutte titanesque entre le vieux pêcheur et un espadon géant. Pourtant, dans le texte en anglais, le poisson n'est pas un espadon, mais un poisson, certes voisin, mais bien distinct, le marlin (marlin en anglais). Pourquoi ce changement de la part du traducteur? Simplement parce qu'il a considéré, à l'époque, 
que le marlin, populaire aux États-Unis, était trop peu connu en France et donc rebuterait le lecteur. Cela serait sans doute encore le cas aujourd'hui. Le passage de marlin en anglais à espadon en français se justifie donc sur le plan de l'accessibilité du texte, mais il s'est fait au prix d'une sérieuse entorse à la vérité zoologique.

\section{Conclusion}

Comme on a pu le voir, la traduction du nom d'un animal est toujours tributaire du type de contexte dans lequel il est situé: document scientifique ou technique, où l'équivalence doit être rigoureuse, texte de vulgarisation, où la précision peut être néfaste, ouvrage littéraire, voire poétique, qui laisse libre cours à toutes les fantaisies...

Les rubriques nécessairement laconiques des dictionnaires usuels, monolingues et bilingues, ne permettent pas toujours de rendre compte de toute la complexité du sujet. Une approche multiculturelle est le plus souvent nécessaire, associant des connaissances de sciences naturelles à des considérations linguistiques. C'est alors en toute connaissance de cause que l'auteur d'une traduction pourra finalement opter pour la dénomination la plus conforme à la réalité scientifique ou pour celle qui se révèle mieux adaptée à un texte plus général, et où la précision zoologique ne s'impose pas.

\section{NOTES}

1. Linné, Carl von (1758): Systema Naturae. 10 édition. Stockholm: Salvius.

2. Gaffiot, Félix (1934): Dictionnaire illustré latin-français. Paris: Hachette.

3. Fishbase - A Global Information System on Fishes. Consultée le 23 juillet 2010, <http://www. fishbase.org>.

4. Walter, Henriette et Avenas, Pierre. Ouvrage sur les noms des poissons, actuellement en préparation.

5. Duval, Alain (1995): Robert \& Collins Super Senior. Paris: Dictionnaires Le Robert.

6. Rondelet, Guillaume (1558): La première partir de l'Histoire entière des Poissons. Avec leurs pourtraicts au naïf. Lyon: Macé Bonhomme; traduction en français, de deux ouvrages publiés par Rondelet en latin: Libri de Piscibus Marinis in quibus verae piscium effigies expressae sunt (1554); Universae aquatilium Historiae pars altera cum veris ipsorum Imaginibus (1555).

7. Collodi, Carlo (1881-1883/1993): Pinocchio. Milan: Feltrinelli. Le commentateur, Fernando Tempesti, cite (note 11, p. 166) ce que Collodi lui-même écrivait dans Gianettino (Collodi 1887: 261): «La balena ha una bocca così spropositata, che può entrarvi comodamente una barca carica d'uomini, ma la sua gola è molto stretta, onde non può ingoiare che piccoli animaletti marini » ( $\mathrm{La}$ baleine a une bouche si disproportionnée qu'il peut y entrer sans difficulté une barque chargée d'hommes, mais sa gorge est très étroite, et elle ne peut avaler que de très petits animaux marins. Traduction des auteurs).

\section{RÉFÉRENCES}

Avenas, Pierre (2005): Dénomination des mammifères dans les langues d'Europe et synonymie. Exposé du 21 octobre 2005 au GEHLF. Sens, Texte, Histoire de Paris IV Sorbonne. Consultée le 20 août 2010, <http://www.sens-et-texte.paris4.sorbonne.fr>.

Dickinson, Edward C. (2003): The Howard and Moore Complete Checklist of the Birds of the World. London: Christopher Helm.

NeLson, Joseph S. (2006): Fishes of the World. New Jersey: John Wiley \& Sons.

WALter, Henriette (2005): Le latin des naturalistes, source d'un bilinguisme bien particulier. La linguistique. 41(2):121-128. 
Walter, Henriette et Avenas, Pierre (2003): L'étonnante histoire des noms des mammifères... De la musaraigne étrusque à la baleine bleue. Paris: Robert Laffont.

Walter, Henriette et Avenas, Pierre (2007): La mystérieuse histoire des noms des oiseaux. Du minuscule roitelet à l'albatros géant. Paris: Robert Laffont.

Wilson, Don E. et Reeder, DeeAnn (2005): Mammal Species of the World. Baltimore: Johns Hopkins University Press. 